\title{
MÉTODO MELHORADO PARA CALCULAR A LEITURA INFINITA DE CINÉTICAS DE PRIMEIRA ORDEM
}

\section{Eduardo Humeres}

Departamento de Química - Universidade Federal de Santa Catarina - 88040-900 - Florianópolis - SC Jairo Quijano

Departamento de Química - Universidad Nacional de Colômbia - Medellín - Colômbia

Recebido em 6/5/96; aceito em 27/10/96

\begin{abstract}
IMPROVED METHOD TO CALCULATE INFINITY READING FOR FIRST ORDER KINETICS. Infinity readings for first order kinetics can be calculated from any three measurements (triads) of a physical property $\lambda$ at three equally spaced times. Accurate results can be obtained from time intervals as low as 0.4 half-life. Calculation of infinity readings $\lambda$ from several triads at increasing values of time gives an insight into the deviation of the first order kinetics when parallel, consecutive or other spurious reactions occur along with the main first order reaction, not allowing direct measurements or calculation of $\lambda$. The proposed method is more sensitive in distinguishing between first and second order kinetics than the Guggenheim and Kezdy-Swinbourne methods.
\end{abstract}

Keywords: infinity reading; kinetic; first-order kinetics.

Cinéticas de primeira ordem são amplamente encontradas em trabalhos experimentais e para calcular a constante de velocidade, usa-se a forma integrada (eq. 1), onde $\boldsymbol{a}$ é a concentração inicial.

$(a-x)=a e^{-k t}$

Quando uma propriedade física $\lambda$ é medida, a eq. 1 torna-se a eq. 2 .

$\lambda-\lambda=\left(\lambda-\lambda_{0}\right) e^{-k t}$

No caso em que $\lambda$ não possa ser obtido, por exemplo em reações extremadamente lentas ou por outras razões, a análise de regressão por mínimos quadrados é aplicada para obter a constante de velocidade, sendo $\lambda, \lambda_{0}$ e $k$ parâmetros ajustáveis destes dados incompletos ${ }^{1}$. No entanto, isto não significa que valores corretos podem ser obtidos ${ }^{2}$. Neste caso, seria conveniente calcular $\lambda$ de $\lambda_{0}$ e $\lambda$, deixando $k$ para ser ajustado de acordo com os dados.

Guggenheim $^{3}$ sugeriu um método para calcular a constante de velocidade de primeira ordem quando a leitura no tempo infinito de uma propriedade física não pode ser obtida (eq. 3)

$\ln \left(\lambda_{2}-\lambda_{1}\right)=-k t_{1}+\ln \left[\left(\lambda_{\times}-\lambda_{0}\right)\left(1-e^{-\mathrm{kD}}\right)\right]$

onde $\mathrm{t}_{2}-t_{1}=\Delta$ é o incremento de tempo constante. O método é usado quando reações consecutivas ou paralelas espúrias ocorrem ao mesmo tempo que a reação principal, mas como não permite calcular $\lambda$ a partir das leituras experimentais, não é possível calcular uma curva de $\lambda$ em função do tempo e comparar com os valores medidos. Além disso, para se obter resultados precisos, o incremento de tempo constante $\Delta$ deve ser no mínimo, igual à meia-vida da reação. Ainda assim, reações de primeira ordem reversíveis e competitivas, e até mesmo reações de segunda ordem, podem dar aparentes constantes de velocidade de primeira ordem ${ }^{2,4}$.

Existem várias situações onde o cálculo prévio de $\lambda$ seria muito prático para estimar-se a meia-vida da reação ou até mesmo para calcular $\lambda$ sem ter-se a leitura infinita experimental. Um exemplo típico onde nós usamos este método é uma reação consecutiva que compete com a reação principal.

Para simplificar-se o cálculo de $\lambda$, vamos considerar três leituras consecutivas $\lambda_{1}, \lambda_{2}, \lambda_{3}$ nos tempos $t_{1}, t_{2}=t_{1}+\Delta$, e $t_{3}$ $=t_{2}+\Delta$, onde $\Delta$ é o incremento de tempo constante. Da eq. 2 , podemos escrever

$$
\begin{gathered}
k=\frac{1}{t_{i}}\left[\ln \left(\lambda_{\infty}-\lambda_{0}\right)-\ln \left(\lambda_{\infty}-\lambda_{i}\right)\right] \\
i=1,2,3
\end{gathered}
$$

$\mathrm{e}$

$\ln \left(\lambda_{\infty}-\lambda_{0}\right)=\frac{t_{2} \ln \left(\lambda_{\infty}-\lambda_{1}\right)-t_{1} \ln \left(\lambda_{\infty}-\lambda_{2}\right)}{t_{2}-t_{1}}=\frac{t_{3} \ln \left(\lambda_{\infty}-\lambda_{2}\right)-t_{2} \ln \left(\lambda_{\infty}-\lambda_{3}\right)}{t_{3}-t_{2}}$

Em consequência, para as três leituras consecutivas, obtémse a eq. 4

$\left\lceil\frac{\left(\lambda_{\infty}-\lambda_{1}\right)^{t 2}}{\left(\lambda_{\infty}-\lambda_{2}\right)^{t_{1}}}\right\rfloor^{\frac{1}{t 2-t 1}}=\left\lceil\frac{\left(\lambda_{\infty}-\lambda_{2}\right)^{t 3}}{\left(\lambda_{\infty}-\lambda_{3}\right)^{t_{2}}}\right\rfloor^{\frac{1}{t 3-t 2}}$

Substituindo-se $\Delta=t_{2}-t_{1}=t_{3}-t_{2}, \quad \lambda$ pode ser calculado a partir de três medidas quaisquer de $\lambda$ a três tempos igualmente espaçados, as quais chamamos tríadas (eq. 5).

$\lambda_{\infty}=\lambda_{1}-\frac{\left(\lambda_{\infty}-\lambda_{1}\right)^{2}}{\lambda_{1}-2 \lambda_{2}+\lambda_{3}}$

O cálculo de $\lambda$ de várias tríadas com valores crescentes de $t$, poderia nos ajudar a visualizar a extensão do desvio das leituras experimentais $\lambda$ dos valores teóricos da cinética de primeira ordem com a possibilidade de usar-se pequenos valores de incrementos de tempo, como se verá abaixo.

Para se analisar os resultados para qualquer valor de $k$, este pode ser expresso em unidades de meia-vida sendo que $k=\ln$ $2 / t_{1 / 2}$ e $k t=\left(t / t_{1 / 2}\right) \ln 2=t^{\prime} \ln 2$, onde $t^{\prime}$ é o tempo medido em unidades de meia-vida, e $\Delta k=\Delta \ln 2 / t_{1 / 2}=\Delta^{\prime} \ln 2$, onde $\Delta^{\prime}$ é o incremento de tempo em unidades de meia-vida. Substituindose $k t$ na eq. 2 a precisão do $\lambda$ calculada pode ser estimada independentemente da velocidade da reação (eq. 6). 
$\lambda=\lambda_{x}+\left(\lambda_{o}-\lambda_{x}\right) e^{-t^{\prime} \ln 2}$

O cálculo de $\lambda$ para $t^{\prime}{ }_{1}=0$ quando $\Delta^{\prime}$ é muito pequeno, requer uma alta precisão da medida de $\lambda$ para obter-se uma leitura infinita precisa, porque o denominador da eq. 5 torna-se muito pequeno. Por exemplo, considerando-se que $\lambda$ possa ser medido com 4 algarismos significativos e $\lambda_{0}=0,000$ e $\lambda=$ 1,000 , se $\Delta^{\prime}=0,2$, a margem de erro de $\lambda$ é de $4 \%$, mas para $\Delta=0,4$ é somente de $1 \%$. Se $\Delta^{\prime}=1$ o erro de $\lambda$ é menor do que $1 \%$ para qualquer valor de $t^{\prime}{ }_{1}$. É óbvio que a escolha de $\Delta^{\prime}$ depende das características da reação.

Cinéticas de segunda ordem do tipo:

$-\frac{d A}{d t}=k_{2} A^{2}$

produzem gráficos lineares razoáveis através do método de Guggenheim ou do gráfico de Kezdy-Swinbourne ${ }^{5,6}$.

Quando uma propiedade física $\lambda$ é medida, a forma integrada da eq. 7 transforma-se na eq. 8, onde $\boldsymbol{a}$ é a concentração inicial.

$\frac{x}{a-x}=\frac{\lambda_{\infty}-\lambda_{0}}{\lambda_{\infty}-\lambda}=a k_{2} \mathrm{t}$

O tempo pode ser expresso em termos de unidades de meiavida considerando-se que $t^{\prime}=t / t_{1 / 2}=a k_{2} t$ (eq. 9).

$\lambda=\frac{\lambda_{0}+t^{\prime} \lambda_{\infty}}{1+t^{\prime}}$

As equações 6 e 9 podem ser utilizadas para comparar cinéticas de primeira e segunda ordem independentemente da meiavida. Nas figs. 1 e 2 são mostrados os gráficos de acordo com Guggenheim e Kezdy-Swinbourne. Notamos que no primeiro caso, até $\mathrm{t}$ ' $=3$, uma reação de segunda ordem produz uma curva com um coeficiente da correlação de 0,992 se a precisão de $\lambda$ for de 4 algarismos significativos. A situação é ainda pior para os gráficos de Kezdy-Swinbourne onde para cinéticas de segunda ordem a curva se aproxima assintoticamente à verdadeira $\lambda$.

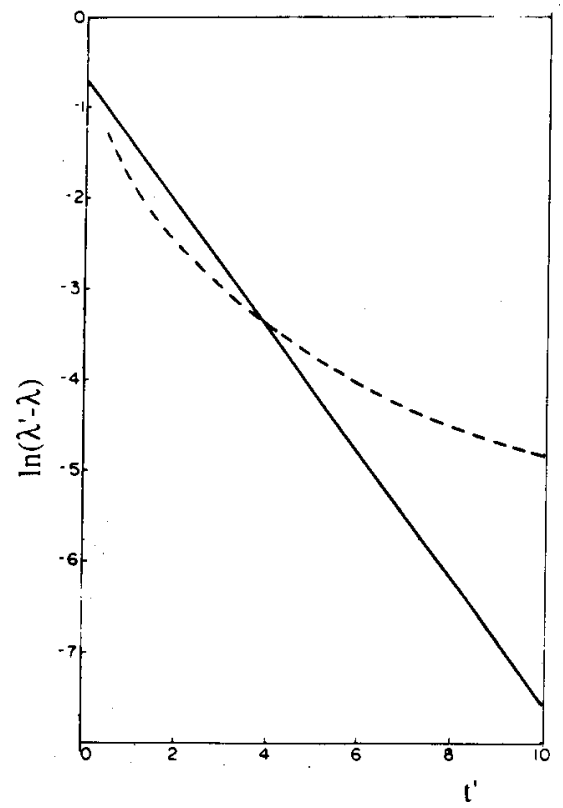

Figura 1. Gráfico de Guggenheim para cinéticas de primeira ( - ) e segunda ordem ( - ) ; $\Delta^{\prime}=1 ; \lambda_{0}=0,000 ; \lambda=1,000$.

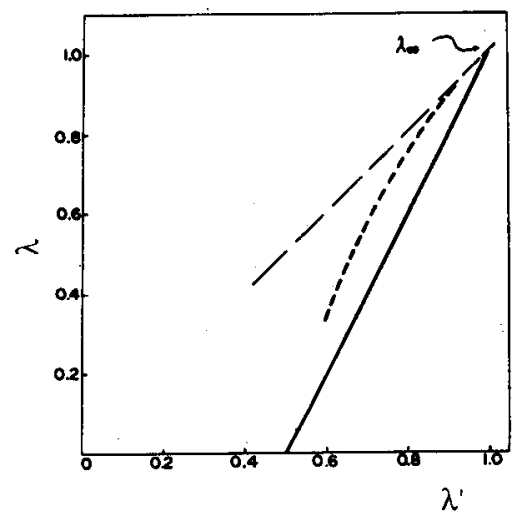

Figura 2. Gráfico de Kezdy-Swinbourne para cinéticas de primeira $(-)$ e segunda ordem $(--), \Delta^{\prime}=1 ; \lambda_{0}=0,000 ; \lambda=1,000$. Reta de tracejado longo calculada para $\lambda=\lambda^{\prime}$.

A mudança de $\lambda$ quando este é calculado pelo método das tríadas para uma cinética de segunda ordem é mostrada na fig. 3. Como $t_{1 / 2}$ depende da concentração inicial, os cálculos foram todos referidos a $t^{\prime}=0$. O $\lambda$ calculado é sistematicamente mais baixo do que o teórico e aumenta uniformemente, dando um diagnóstico claro de que a cinética não é de primeira ordem.

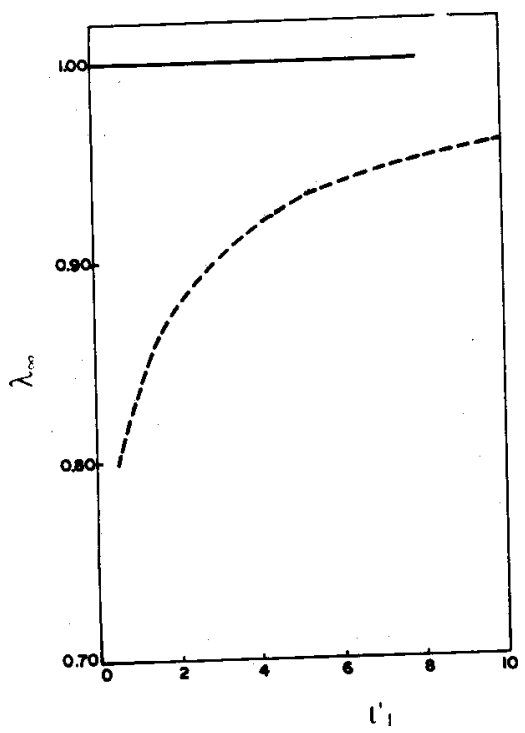

Figura 3. Leituras a tempo infinito calculadas através do método das tríadas para cinéticas de primeira ( - ) e segunda ordem (- - ); $\Delta^{\prime}=$ $1, \lambda_{\mathrm{o}}=0.000 ; \lambda=1,000$.

\section{REFERÊNCIAS}

1. Wiberg, K.; Use of Computers; In Investigation of Rates and Mechanisms of Reactions, Part I; Lewis, S. E., Ed.; In Techniques in Chemistry, Weissberger, A., Ed.; Wiley; New York, 1974; vol. 6.

2. Moore, J. W.; Pearson, R. G.; Kinetics and Mechanism; Wiley; New York, 1981; Cap. 3.

3. Guggenheim, E. A.; Phil. Mag. 1926, 2, 538.

4. Swinbourne, E. S.; Analysis of Kinetic Data; Nelson; London, 1971; Cap. 4.

5. Kezdy, F. J.; Jaz, J.; Bruylants, A.; Bull. Soc. Chim. Belg. 1958, 67, 687.

6. Swinbourne, E. S.; J. Chem. Soc. 1960, 2371. 